\title{
Prácticas de equidad y calidad en la Universidad Veracruzana, Campus Poza Rica
}

\author{
MARILÚ VILLALOBOS LÓPEZ \\ Universidad Veracruzana Región Poza Rica - México \\ ADORACIÓN BARRALES VILLEGAS ${ }^{* *}$ \\ Universidad Veracruzana Región Poza Rica - México \\ REGINA DAJER TORRES ${ }^{* * *}$ \\ Universidad Veracruzana Región Poza Rica - México \\ Recibido el 12-11-18; primera evaluación el 06-05-20; \\ segunda evaluación el 21-07-20; aceptado el 25-08-20
}

\section{Resumen}

A pesar de los avances que la humanidad ha tenido en diferentes esferas, todavía no se ha logrado garantizar de manera universal los derechos humanos o brindar calidad de vida a toda la humanidad. Un aspecto que se vuelve clave es la equidad, pues cuando la ausencia de equidad se presenta en una institución educativa, se ve afectada también su calidad. Por tal motivo, el presente escrito muestra resultados obtenidos de una investigación sobre Equidad y Calidad como parte del Proyecto

\footnotetext{
* Doctora en Medición Pedagógica. Profesora de Tiemplo Completo y tutora académica en la Facultad de Pedagogía, de la Universidad Veracruzana Región Poza Rica, México. Integrante del Cuerpo Académico Gestión e Investigación Educativa Transdisciplinaria (GIET). Cuenta con reconocimiento de Perfil Deseable PROMEP por parte del Comité Evaluador externo SEP/ Subsecretaria de Educación Superior, México. Directora de tesis a nivel licenciatura y maestría. Correo electrónico: marilu_vl@ hotmail.com. https://orcid.org/0000-0003-1956-7456

** Licenciada en Pedagogía. Magíster en Educación. Doctora en Calidad y Procesos de Innovación Educativa. Profesora de tiempo completo titular "C» de la Universidad Veracruzana. LGAC: Orientación Educativa y Sustentabilidad; Didáctica y Evaluación. Miembro del Sistema Nacional de Investigadores (SNI), nivel candidato del Consejo Nacional de Ciencia y Tecnología (CONACYT). Perfil Deseable PROMEP por parte del Comité Evaluador externo SEP/ Subsecretaria de Educación Superior, México 2011 y refrendos en 2014 y 2017. Correo electrónico: abarrales@uv.mx. https:// orcid.org/0000-0003-1880-9169

${ }^{* * *}$ Profesora de Tiemplo Completo en la Facultad de Pedagogía, de la Universidad Veracruzana. Integrante del Cuerpo Académico Gestión e Investigación Educativa Transdisciplinaria (GIET), en la línea de investigación Orientación Educativa y Sustentabilidad. Cuenta con reconocimiento de Perfil Deseable PROMEP por parte del Comité Evaluador externo SEP/ Subsecretaria de Educación Superior. Directora de tesis a nivel licenciatura y maestría. Tiene el grado de doctora. Correo electrónico: rdajer@uv.mx. https://orcid.org/0000-0002-6158-7728
} 
Europeo ORACLE 2019 (Observatorio Regional de Calidad de la Equidad de la Educación Superior en Latinoamérica), realizada en la Universidad Veracruzana en su Región Poza Rica - Tuxpan. Para este informe, se presentan específicamente los resultados obtenidos en la Facultad de Arquitectura. Dicha investigación pretendía conocer las prácticas de equidad y calidad que se observan en dicha institución desde la perspectiva del personal docente y de los estudiantes.

Palabras clave: universidad, equidad, calidad.

\section{Equity and quality practices at the Universidad Veracruzana, Campus Poza Rica}

\section{Abstract}

Despite the progress that humanity has made in different spheres, it has not yet been possible to guarantee universal human rights or providing quality of life to all of humanity. An aspect that becomes key is equity and, when the absence of equity occurs in an educational institution, its quality is also affected. For this reason, the following article shows the results obtained from a research on Equity and Quality as part of the European Project ORACLE 2019 (Regional Observatory of Quality of Equity or Higher Education in Latin America) carried out at the Universidad Veracruzana in its Poza Rica - Tuxpan Region. This report specifically presents the results obtained in the Faculty or Architecture. The objective of this research was to understand the practices of equity and quality that can be observed in said institution from the perspectives the teaching staff and students.

Keywords: university, equity, quality.

\section{Práticas de equidade e qualidade na Universidad Veracruzana, Campus Poza Rica}

\section{Resumo}

Apesar do progresso que a humanidade fez em diferentes esferas, ainda nao foi possível garantir direitos humanos universais ou proporcionar qualidade de vida a toda a humanidade. Um aspecto que se torna fundamental é a eqüidade e, quando a ausencia de eqüidade ocorre em uma instituicao de ensino, suas qualidades também é afectada. Por esse motivo, esrte artigo mostra os resultados obtidos em uma investigacao sobre Equidade e Qualidade como parte do Projeto Europeu ORACLE 2019 (Observatório Regional de Qualidade de Equidade do Ensino Superior na América Latina) realizado na Universidad Veracruzana em sua Regiao Poza Rica - Tuxpan. Para este relatório, apresenta específicamente os resultados obtidos na Faculdade de Arquitetura. Esta pesquisa teve como objetivo conhecer as prácticas de equidade e qualidade observadas na referida instituicao na perspectiva do corpo docente e dos alunos.

Palavras-chave: universidade, equidade, qualidade. 


\section{INTRODUCCIÓN}

Sin lugar a duda, «la calidad de la educación se ha convertido en las sociedades de nuestro tiempo en un tema estrella...» (Santos, 2013, p. 21). La educación actual está centrando sus esfuerzos en la promoción de prácticas de equidad orientadas a garantizar la calidad de las instituciones, sin embargo no se puede hablar de calidad cuando no se garantiza la equidad en la educación. La misma Unesco (2017), en su Informe de Seguimiento de la Educación en el Mundo 2016, contiene la Meta 4.1 Equidad, lo cual deriva de la necesidad de garantizar una educación de calidad, equitativa e inclusiva para todos.

Lo anterior invita a voltear la mirada hacia las prácticas de equidad que se viven diariamente en todos los ámbitos y, en especial, el educativo. Desde esta perspectiva, hablar de equidad en el ámbito educativo conlleva al abordaje de una serie de costumbres y posturas paradigmáticas que en ocasiones generan injusticia entre los integrantes de la comunidad educativa. Lo cual implica que, de manera directa o indirecta, las estrategias de equidad que se utilizan en una institución educativa dependen de la propia conceptualización que se tenga sobre lo que significa el concepto de equidad.

Según Hopenhayn en NU. CEPAL OEA (1995), «la equidad deja de entenderse como acceso 'genérico' a la educación formal, y empieza a definirse según la pertinencia de la educación a la cual se tiene acceso» (p. 41). Cabe mencionar que, desde nuestra perspectiva, la equidad supone que se proporcionen no solo los recursos sino las circunstancias para brindar las mismas oportunidades para que todos estén en posibilidad de alcanzar las metas educativas deseables.

Por tal motivo, realizamos un análisis institucional encaminado a conocer las prácticas y políticas de equidad existentes en la Universidad Veracruzana en su Región Poza Rica - Tuxpan. A fin de contextualizar, mencionaremos que la Universidad Veracruzana (U.V.) es la máxima casa de estudios a nivel estatal debido a que «cuenta con cinco sedes regionales: Xalapa, Veracruz, OrizabaCórdoba, Poza Rica - Tuxpan y Coatzacoalcos - Minatitlán con presencia en 27 municipios. Por su matrícula, se ubica entre las cinco universidades públicas estatales de educación superior más grandes de México» (Universidad Veracruzana, 2020, Sección de Presentación, párrafo 1). Dicha universidad, preocupada por brindar una educación de calidad, intenta eliminar las desigualdades a través de la promoción de políticas generadoras de prácticas de equidad entre su comunidad. Además, cuenta con una normativa encaminada a garantizar equidad, entre las cuales podemos citar la Ley Orgánica de la Universidad Veracruzana (2017) bajo la que se rige la institución, el 
Reglamento de la Defensoría de los Derechos Universitarios, el Estatuto General, y el Código de Ética, entre otros reglamentos.

Dado los esfuerzos que la U.V. realiza en materia de equidad y en el alcance de la calidad educativa, se realizó el análisis en las diferentes Facultades de la Región Poza Rica - Tuxpan, siendo un total de 15 facultades encuestadas; sin embargo, en este informe presentamos solamente los resultados obtenidos en la Facultad de Arquitectura en su Campus Poza Rica. Cabe mencionar que la población encuestada estuvo conformada por 72 alumnos de periodos escolares variados y 8 docentes. En el apartado de metodología explicamos cómo se decidió la muestra.

Los objetivos de investigación son los siguientes:

- Elaborar un diagnóstico para conocer la percepción que los integrantes tienen del desarrollo de equidad.

- Analizar la importancia que los integrantes dan a la equidad dentro de los procesos institucionales.

- Identificar las áreas de oportunidad para mejorar las prácticas institucionales en el manejo de equidad.

- Identificar la percepción de los integrantes en el manejo de la política de equidad institucional y su contribución para mejorar la calidad educativa.

- Describir las buenas prácticas que se desarrollan en el alcance del principio de equidad.

\section{MarCo teórico}

Como mencionamos anteriormente, hablar de calidad obliga indudablemente a voltear la mirada a nuestras prácticas de equidad, pues estas impactan no solo los procesos educativos de una institución educativa sino también los administrativos, sociales, culturales, etc. Por tal razón, coincidimos con Gairín y Suárez (2018) cuando exponen que «...el principio de equidad y la atención a la diversidad no pueden desconocer la búsqueda de la calidad en la educación» (p. 33).

Dada la trascendencia de ambos conceptos, procedemos a aproximarnos a ellos con la intención de comprender su esencia. Iniciamos con calidad en la educación, la cual según esta opinión:

Es un paradigma que pretende desplazar el pensamiento cartesiano, lineal, ingenieril tradicional, por otro distinto, por uno complejo, sistémico, transdisciplinar 
que muestre el mundo a los estudiantes de otra forma, donde lo disjunto se supere y se comprenda la unidad del planeta en que vivimos. (Yanes, 2015, p. 46)

Por su parte, esta otra perspectiva considera que:

...la calidad, al menos en lo que se refiere a las escuelas, no es tanto un repertorio de rasgos que se poseen, sino más bien algo que se va consiguiendo. La calidad es algo dinámico (por eso se alude más a las condiciones culturales de las escuelas que a sus elementos estructurales), algo que se construye día a día y de manera permanente. (Zabalza, 2016, 47)

En este sentido,

...la Organización para la Cooperación y Desarrollo Económico (OCDE), organización que agrupa a treinta países europeos y americanos — los más desarrollados del planeta-, en la que los ministros de educación dieron a conocer a la opinión pública que la calidad de la educación sería una de las máximas prioridades de las siguientes décadas (Santos, 2013, p. 22).

Es un aspecto que en nuestro país y, específicamente en la universidad objeto de estudio, se ha estado observando ya sea en adecuaciones de infraestructura, adecuaciones curriculares, políticas de calidad, etc.

Ahora bien, como ejemplo de lo que se hace fuera de nuestro país, la ONG Educación 2020 (fundación chilena) también pone cartas sobre el asunto en materia de calidad en la educación:

E2020 plantea siete principios básicos que tampoco se separan de lo que plantea la Unesco y la OCDE para la educación del siglo que estamos comenzando. Entre estos principios está que el Estado debe asegurar calidad en la educación, pero entiende "calidad» solo como el aprendizaje integral de saberes y habilidades desde los primeros ańos de vida hasta la educación superior (Yanes, 2015, p. 68).

Por otra parte, en lo que se refiere a «equidad» consideramos necesario comentar que, en nuestro contexto universitario, somos conscientes de que no todo el que desea ingresar lo logra. En palabras de Joaquín Gairín, «... la Educación Superior (ES) sigue estando vetada a grupos sociales desfavorecidos...» (Gairín, Castro, Rodríguez y Barrera, 2015, p. 11). Ante tal situación, "la equidad podría, entonces, ser una práctica indispensable para llegar al ideal de justicia y de ciudadanía plena, capaces de garantizar el goce de una situación de igual bienestar para todos los ciudadanos» (Ricaurte et al., 2018, p. 156). 
Además, «despreciar las diferencias provoca discriminación, pues se convierten en límites y obstáculos» (Calvo, 2011, p. 25) para garantizar la equidad a la comunidad universitaria, de modo que un elemento clave para la generación de acciones a favor de la equidad es que tanto los estados como las instituciones cuenten con normatividad y políticas reguladoras de la misma.

En nuestro país, México, se han realizado diversas acciones al respecto. Por ejemplo, en 2017 se presentó un Nuevo Modelo Educativo (NME) que contempla ejes rectores: «el cuarto [eje] es la inclusión y la equidad. Siguiendo el enfoque humanista, el modelo reconoce la diversidad que existe entre alumnos y escuelas, así como la interculturalidad de la educación» (Granados, 2018, p. 78).

Ahora bien, si consideramos que «la educación de buena calidad no debe ser equiparada, o reducida, a los resultados del aprendizaje» (Unesco, 2017), entonces las instituciones educativas deberían centrarse en brindar un servicio equitativo y de calidad. Por ello, coincidimos con Llach (2010) cuando expresa que «...una verdad de a puño, como diría Sarmiento, que el acceso igualitario a una educación de calidad depende crucialmente del nivel de vida y de la equidad de cada sociedad» (p. 11). Es decir, «...procurar una relación entre las oportunidades educacionales y evitar las desigualdades sociales, especialmente en lo que se refiere a sus causas y consecuencias, procurando establecer una correspondencia entre los problemas sociales, étnicos, raciales y de género» (Flacso, 2017, p. 197).

Luego entonces, «...cerrar las brechas de calidad de la oferta educativa que imparten las instituciones representa un objetivo estratégico en el propósito de lograr la equidad» (Rubio y Mungaray, 2009, 159), aspecto clave en la presente investigación.

\section{Metodología}

En cuanto a la metodología utilizada para la recogida de la información, fue cuantitativa toda vez que nos interesaba conocer de manera detallada aspectos medibles del fenómeno de estudio. Según Hernández (2010), en una investigación cuantitativa se «...intenta explicar y predecir los fenómenos investigados buscando regularidades y relaciones causales entre los elementos... nos conduce a una explicación sobre cómo se concibe la realidad...» (p. 6).

Nos decidimos por un tipo de estudio descriptivo de encuesta segmentada aplicada a una muestra de la población de la Universidad Veracruzana en su Región Poza Rica - Tuxpan. Cabe mencionar que los estudios descriptivos 
«permiten organizar y clasificar los indicadores cuantitativos obtenidos en las investigaciones empíricas, determinando a través de ellas las propiedades, relaciones y tendencias del fenómeno» (Hernández y Coello, 2008, p. 94). Como nos interesaba analizar la percepción que dicha población tiene sobre el manejo de equidad, de cada Facultad se decidió encuestar el 30\% de la población estudiantil. Para el caso de la Facultad de Arquitectura, en el periodo de aplicación (febrero - julio 2019) contaba con un total de 208 alumnos, de los cuales 99 eran mujeres y 109 hombres. El 30\% fue superado a 34,6\% de la población debido a la respuesta obtenida por el estudiantado. En palabras de Hernández et. al. (2010), "en una investigación cuantitativa se pretende generalizar los resultados encontrados en un grupo o segmento (muestra) a una colectividad mayor (universo o población)» (p. 6.).

Nuestro estudio posee un corte transversal debido a que su realización fue solo en el periodo agosto 2017 - enero 2018. En cuanto al instrumento utilizado para la encuesta, creemos necesario mencionar que este nace de la propuesta de un grupo de docentes que forman parte del Observatorio de Equidad y Calidad de la Educación Superior (ORACLE). Tal instrumento está compuesto de 43 ítems de opción múltiple con base en una escala de Likert y se integró por tres apartados para su análisis:

- Pertinencia de las políticas institucionales

- Detección de necesidades y problemáticas

- Opiniones de los encuestados con respecto a la operatividad de la normativa, promoción de la equidad en la institución, estrategias que se implementan y el desarrollo de la cultura de la calidad educativa

\section{Resultados}

Una vez procesada la información recabada a través de la encuesta, pudimos obtener los siguientes resultados en relación con el primer apartado, Pertinencia de las Políticas Institucionales:

Tabla 1. Promoción de las políticas de equidad: docentes

\begin{tabular}{lc}
\hline \multicolumn{1}{c}{ Muy importante } & $50 \%$ \\
\hline Importante & $50 \%$ \\
Indiferente & $0 \%$ \\
Poco importante & $0 \%$ \\
\hline
\end{tabular}

Fuente: Elaboración propia con base en los resultados. 
Figura 1. Pertinencia de las políticas de equidad: estudiantes

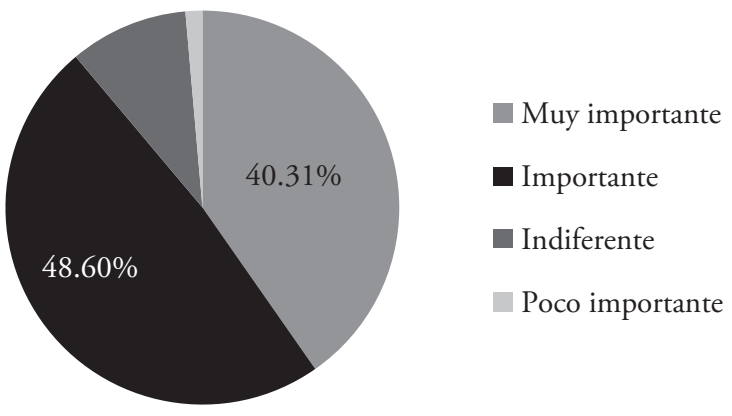

Fuente: Elaboración propia con base en los resultados.

Como se puede observar, el personal docente de la Facultad de Arquitectura tiene apertura hacia la equidad. En lo que respecta a los estudiantes, si sumamos las respuestas que están a favor en nivel de «importante» y en «muy importante», obtenemos un $88,91 \%$ de los estudiantes encuestados que coinciden en que es necesariamente importante la promoción de políticas de equidad en la Facultad de Arquitectura de la Universidad Veracruzana.

Cuando se les interrogó sobre el manejo de la equidad institucional con respecto a la calidad de la educación que se imparte en la U.V., el 75\% de los docentes encuestados relacionan la equidad con la calidad de la institución (ver tabla 2).

Tabla 2. Manejo de la equidad institucional con respecto a la equidad: docentes

\begin{tabular}{lc}
\hline \multicolumn{1}{c}{ Muy importante } & $50 \%$ \\
\hline Importante & $25 \%$ \\
Indiferente & $12,5 \%$ \\
Sin responder & $12,5 \%$ \\
\hline
\end{tabular}

Fuente: Elaboración propia con base en los resultados. 
Figura 2. Manejo de la equidad institucional con respecto a la calidad de la educación que se imparte en la U.V.

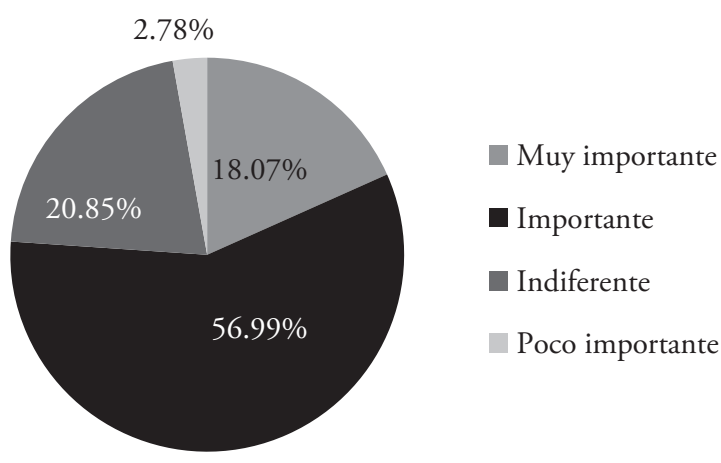

Fuente: Elaboración propia con base en los resultados.

En lo que respecta al alumnado, la mayoría opinó que el manejo de la equidad con respecto a la calidad de la educación en la U.V. es una necesidad. No obstante que la mayoría está a favor del manejo de equidad, un $20.85 \%$ se muestra indiferente al respecto y un $2.78 \%$ incluso lo considera poco importante (Fig. 2).

Tabla 3. ¿Existen diferencias en los términos equidad e igualdad? Docentes

\begin{tabular}{lc}
\hline Son diferentes & $50 \%$ \\
Parcialmente diferentes & $50 \%$ \\
\hline Fuente: Elaboración propia con base en los resultados.
\end{tabular}

Un aspecto importante de resaltar surgió cuando los cuestionamos sí consideraban que existían diferencias entre los términos de equidad e igualdad. Se puede observar que los docentes argumentan que ambos conceptos son diferentes en su totalidad, aspecto que permite inferir que para ellos equidad e igualdad no son sinónimos o que dichos conceptos son parcialmente diferentes, pero no queda claro en qué o por qué los consideran así.

Ahora bien, un número significativo de estudiantes considera sinónimos a la equidad e igualdad toda vez que el 13,9\% opinó que para nada existen diferencias entre ambos términos. Por su parte, un 33,36\% opina lo contrario, pues expresan estar totalmente de acuerdo en que ambos conceptos son totalmente diferentes. Lo anterior se ve confirmado en sus respuestas a la siguiente interrogante, donde se observa que un $8,34 \%$ cree que no existe relación 
alguna entre equidad y calidad educativa mientras que un 50,04\% considera totalmente vinculados ambos términos (ver figura 3).

Tabla 4. Relación entre equidad y calidad educativa

\begin{tabular}{ll}
\hline Existe una relación total & $25 \%$ \\
Existe una relación parcial & $50 \%$ \\
No existe relación alguna & $25 \%$ \\
\hline
\end{tabular}

Fuente: Elaboración propia con base en los resultados.

Figura 3. Relación entre equidad y calidad educativa

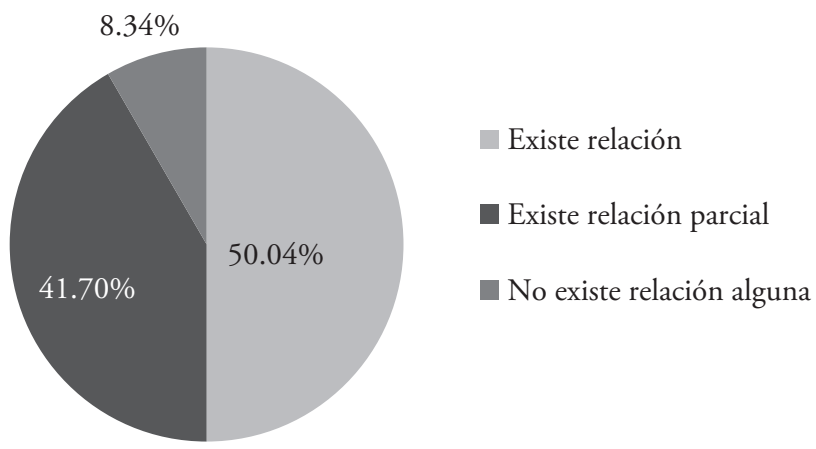

Fuente: Elaboración propia con base en los resultados.

Otro elemento a investigar fue si consideraban que la equidad está relacionada con la calidad educativa. La mayoría de la población docente encuestada considera que es una relación parcial o total con la calidad educativa; sin embargo, un $25 \%$ opina que dicha relación se da "totalmente» y el $25 \%$ restante cree que para «nada se relacionan». $\mathrm{Al}$ analizar las respuestas se observa que se contradicen en la interrogante número 2 , pues en ella la mayoría consideraba importante dicha relación, aspecto que ahora niegan o desconocen. Lo anterior permite observar que no hay claridad de que la equidad es un indicador de calidad educativa en una institución, es decir, que cuando una institución educativa no garantiza equidad a sus estudiantes afecta la calidad de sus servicios. En cuanto a los alumnos, un número significativo manifiesta que existe relación ya sea total o parcial entre ambas. 
Tabla 5. La universidad cuenta con políticas pertinentes en el manejo de equidad

\begin{tabular}{ll}
\hline Sí cuenta & $62,5 \%$ \\
Cuenta parcialmente & $25,0 \%$ \\
Para nada cuenta & $12,5 \%$ \\
\hline
\end{tabular}

Fuente: Elaboración propia con base en los resultados.

Figura 4. La universidad cuenta con políticas pertinentes en el manejo de equidad

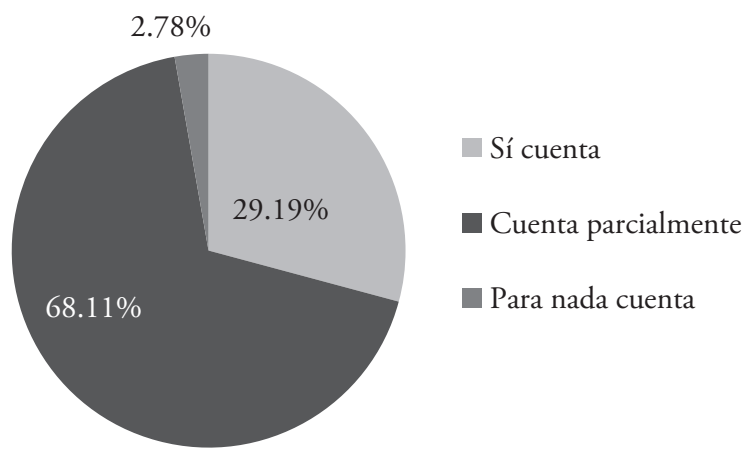

Fuente: Elaboración propia con base en los resultados.

En cuanto a si consideraban que la universidad tiene políticas institucionales pertinentes para el manejo de equidad, la mayoría de los docentes encuestados opinan que la Universidad Veracruzana sí cuenta con políticas institucionales pertinentes en el manejo de equidad, mientras que un número significativo denota un desconocimiento de las políticas y normatividad relativas al manejo de la equidad existentes en la U.V. Por parte de los estudiantes, un porcentaje representativo (aproximadamente un 70\%) consideran que las políticas institucionales con relación a equidad son pertinentes. Solo un $2,78 \%$ cree que no son pertinentes las políticas existentes en el manejo de equidad al interior de la U.V. 
Figura 5. Conocimiento sobre la política institucional en materia de equidad: alumnos

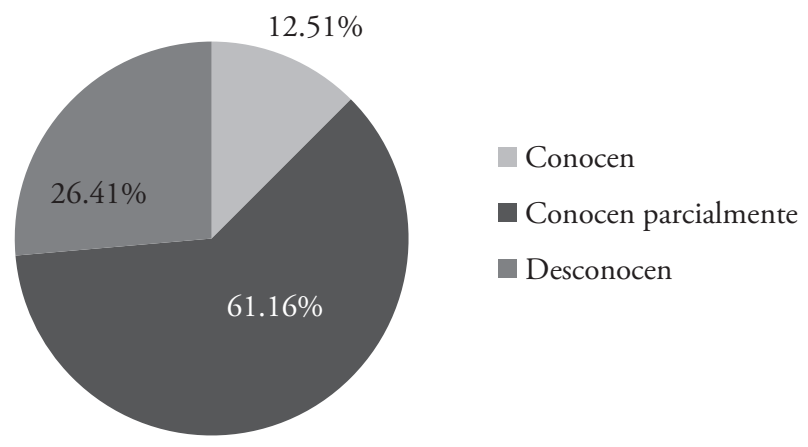

Fuente: Elaboración propia con base en los resultados.

También se les cuestionó sobre si conocían la política institucional en materia de equidad, a lo que un $25 \%$ de los académicos encuestados aceptaron que para nada conocen la política institucional en materia de equidad, 37,5\% asegura conocerla parcialmente y solo el $37,5 \%$ afirma conocer en su totalidad la política institucional en el manejo de equidad. En lo que se refiere al otro estamento (ver figura 5), solo el $12,51 \%$ de la población estudiantil encuestada expresa conocer dicha política institucional. Lo anterior se relaciona de manera porcentual con el hecho de que solo el $16,68 \%$ conocía a los responsables en materia de equidad, es decir, el desconocimiento de los responsables coincide con el desconocimiento de la política institucional.

Respecto a si aplica equidad en su ámbito de competencia, el $75 \%$ de la población docente encuestada asegura que aplica la equidad en su totalidad en sus ámbitos de competencia, mientras que el $25 \%$ restante afirma que también lo hace, pero de manera parcial. En cuanto a si los estudiantes aplican o no equidad en sus ámbitos de competencia, pudimos observar que un 52,.82\% de los encuestados respondieron que sí la aplican en su totalidad. Un significativo $43,09 \%$ asegura aplicar equidad de manera parcial. Finalmente, un 4,17\% de los encuestados aseguran que para nada aplican equidad en sus ámbitos. Ahora bien, la manera en que algunos de ellos expresaron que aplican equidad se muestra a continuación:

- En la práctica profesional

- Mismo trato para todos

- Al participar en las actividades académicas

- Al respetar la normativa 
- Dando la misma importancia a todos

- Teniendo las mismas oportunidades

En lo que respecta al segundo apartado, detección de necesidades y problemáticas, será reportado de manera separada. Iniciamos presentando las necesidades detectadas: el género, los escasos recursos económicos, los problemas de salud, la edad y el lugar de procedencia son las condiciones mencionadas por los docentes que les ha puesto en desventaja alguna vez; sin embargo, cabe aclarar que solo fue el 12,.5\% de los docentes encuestados quienes opinaron de esa manera, es decir, una minoría. La mayoría de ellos asegura que nunca se han visto en desventaja por los motivos aquí citados. Cabe mencionar que la gran mayoría de los estudiantes opinaron que nunca se han sentido en desventaja derivada de las condiciones enlistadas; por ejemplo, en lo referente a género, un 73,67\% expresó que nunca se han visto en desventaja por esta cuestión, sin embargo un 25,02\% aceptó que algunas veces se han visto en situación de y un 1,39\% aseguró que siempre se siente en desventaja, sobre todo porque cuando ofrecen trabajo dan preferencia a los varones. El origen étnico es otra condición que un 1,39\% menciona que siempre lo coloca en desventaja, mientras que un 6,95\% también acepta que el origen étnico le pone en desventaja pero solo algunas veces. Por el contrario, el $91,74 \%$ asegura que nunca les ha afectado. La edad se señala como que siempre afecta al $2,78 \%$ de los encuestados, contrario a lo que un gran $83,4 \%$ de dicha población niega sufrir pues aseguran que nunca les ha puesto en situación de desventaja. En cuestión de los escasos recursos económicos o pobreza hubo un $26,41 \%$ que aceptó verse afectado en algunas ocasiones, aunque la gran mayoría, es decir, un 73,67\% niegan que sea una condición que les haya afectado.

Al parecer la Facultad de Arquitectura carece de problemáticas en la forma en que se relacionan toda vez que solamente el 12,5\% (una persona) de los docentes encuestados aceptan que siempre hay problemas en la forma de relacionarse entre alumno y secretaria, mientras que contradictoriamente el 50\% dice que nunca se presentan problemas entre alumnos y secretarias. La mayoría coincidió en que nunca se presentan problemas de este tipo; por ejemplo, el $75 \%$ asegura que nunca se presentan casos de discriminación al interior de la institución, el $62,5 \%$ de ellos dice que nunca se suscitan problemas de acoso, hostigamiento o maltrato verbal. Por su parte, el $50 \%$ de los docentes exponen que nunca existen problemáticas tales como prepotencia/abuso del poder o problemáticas de las relaciones entre maestros. 
En cuanto a la forma de relacionarse entre maestros y personal administrativo, por una parte, y alumno con alumno por otra, los entrevistados (un $37,5 \%)$ aseguran que nunca se presentan este tipo de problemáticas. Cabe resaltar que algunos docentes prefirieron optar por la opción del desconocimiento toda vez que un significativo $37,5 \%$ asegura no saber si existen problemas en la forma de relacionarse entre maestros y directivos. Solo un $25 \%$ reconoce que algunas veces existen problemáticas entre maestros y personal administrativo.

En cuanto a alumnos, la prepotencia y el abuso de poder es la problemática que en mayor porcentaje se mencionó por los estudiantes de la Facultad de Arquitectura como presente en la institución, con un 9,73\%, apoyado por un $23,63 \%$ que acepta que la problemática se ha presentado algunas veces; sin embargo, un $44,48 \%$ de los estudiantes expresa que nunca se han dado este tipo de problemáticas en la institución y un 16,68\% comentó que casi nunca se presentan tales situaciones. Si hacemos un análisis de los datos, podemos observar que existen problemáticas de abuso de poder y prepotencia en dicha Facultad toda vez que ya sea en mayor o menor medida, el $50,04 \%$ reconoce que se han presentado. Problemáticas de relaciones entre maestros y alumnos fue reconocida por un $6,95 \%$ con una frecuencia de siempre presente en Arquitectura, seguida de un 23,63\% que argumenta que algunas veces se presentan este tipo de problemáticas y un 9,73\% dice que casi nunca se suscitan pero que sí se dan. Por el contrario, el 43,09\% expone que nunca se dan problemas en la forma de relacionarse entre maestros y alumnos. En relación al acoso, un 63,94\% de los estudiantes comentaron que nunca se presenta este tipo de problemática en la institución, no obstante, un 8.34\% acepta que sí se presenta acoso pero casi nunca sucede. Si agregamos que un $6.95 \%$ opina que algunas veces existe acoso y otro 5,56\% acepta que siempre se presentan casos de acoso en Arquitectura. El maltrato verbal es reconocido por un $19,46 \%$ como una problemática que algunas veces se presenta en la institución; en este sentido coincide con el 4,17\% que asegura que siempre se presenta, mientras que un $12,51 \%$ acepta que casi nunca suceden casos de maltrato verbal pero que sí se presentan. Contrariamente, el 55,6\% de los estudiantes encuestados opinó que nunca se presenta este tipo de problemática en Arquitectura.

Finalmente, en cuanto al tercer apartado "Opiniones de los encuestados con respecto a la operatividad de la normativa, promoción de la equidad en la institución, estrategias que se implementan y el desarrollo de la cultura de la calidad educativa» pudimos observar que el $50 \%$ de los docentes encuestados 
considera que en la Facultad de Arquitectura siempre son apropiadas las prácticas de equidad, el 25\% dice que lo son pero solo algunas veces y el 12,5\% expone desconocer si son o no apropiadas dichas prácticas. En cuanto a si las prácticas de equidad se desarrollan exitosamente, el 50\% de los docentes afirman que así es, un $12,5 \%$ asegura que solo algunas veces son exitosas, otro $12,5 \%$ dice que casi nunca son exitosas mientras que otro $12,5 \%$ asegura desconocerlo. Es necesario comentar que un docente omitió participar en este apartado. Como se pudo observar, el 33,36\% de los jóvenes asegura que siempre y el 43,09\% que algunas veces son apropiadas las prácticas de equidad en la Facultad de Arquitectura, mientras que un 1,39\% asegura que dichas prácticas nunca lo son. Al revisar las razones del por qué lo consideraban de esa manera encontramos que los chicos:

- Se encontraban a favor: porque todos son tratados igual, buscan lo mejor para la población estudiantil, son inclusivos, todos trabajamos en equipo, pues todo es igual con todos, porque existe respeto, porque todos somos iguales, porque todos respetamos a todos, a todos nos tratan por igual.

- Se encontraban en desacuerdo: porque el contexto no ayuda mucho para personas discapacitadas, aunque se hable de ellos; podrían emplear más; por las actividades realizadas.

En cuanto a la importancia de que en la Universidad Veracruzana se promuevan y cultiven valores, el $87,5 \%$ de los profesores encuestados coincide en que es muy importante fomentar en la U.V. los valores de tolerancia, tespeto a la diversidad de ideas, dignidad, igualdad y no discriminación, y libertad y responsabilidad. Con un $75 \%$ de coincidencia en muy importante le siguen los valores de ética profesional, compromiso social, democracia, seguridad y cuidado, honestidad e integridad, transparencia y rendición de cuentas, y equidad. Por su parte, el 62,5\% de la población encuestada opina que es muy importante fomentar los valores de respeto a la diversidad sexual, disposición, solidaridad, e imparcialidad, objetividad, independencia. Cabe mencionar que ninguno de los encuestados manifestó como nada importante, poco importante o indiferente la promoción de los valores enlistados. Por su parte, el 63,8\% de los alumnos coinciden en elegir a la equidad como el valor más necesario de cultivar, en segundo término, citan a la honestidad e integridad, ética profesional y respeto a la diversidad de ideas con un $62,5 \%$ de menciones y en tercer lugar con un $61,11 \%$ los valores dignidad, igualdad y no discriminación, así como la libertad y responsabilidad. 
En relación a qué actitudes ayudarían a promover la calidad del programa de Arquitectura, los docentes expusieron en un $87,5 \%$ que la colaboración y el compromiso hacia la calidad del trabajo eran muy importantes en la consecución de esa meta. Con un 75\% le siguen actitudes como disposición, receptividad crítica y apertura al diálogo. El 62,5\% coincide en que proactividad es una actitud que permitiría de manera muy importante de ayudar a promover la calidad. Mientras que un 50\% asegura que también son muy importantes actitudes como solidaridad y cordialidad. Finalmente, un 37,5\% también considera muy importante la actitud de empatía. Cabe aclarar que ningún docente opinó «indiferencia», "poco» o «nada» de importancia hacia las actitudes enlistadas. Por su parte, el 55,55\% de los alumnos encuestados que la proactividad y la receptividad crítica son actitudes importantes para promover y el 54,16\% valoran el compromiso hacia la calidad del trabajo. Actitudes de disposición son las siguientes en mención de parte del estudiantado, con un $52,7 \%$ y un $51,8 \%$. Cabe mencionar que un $11,11 \%$ se manifiestan indiferentes frente a actitudes como la empatía y un $8,33 \%$ a la proactividad y la cordialidad.

Cuando les solicitamos mencionar actitudes que observan en su Facultad donde se desarrolla la equidad, el personal académico expresó las siguientes:

- Actitud positiva generosa

- Respeto

- Tolerancia

- El trato equitativo a los alumnos, al personal y a los maestros

Los alumnos, por su parte, mencionaron las siguientes actitudes que observan en su Facultad donde se desarrolle la equidad:

- Respeto

- Al ayudarnos

- Cuando no hay preferencias

- Igualdad

- Tolerancia

- Compañerismo

- Solidaridad

- Amabilidad

- Disposición

- Empatía

- Democracia

- Unión 
Un elemento interesante para resaltar surgió cuando les solicitamos actitudes que observan donde se evidencia la falta de equidad. El personal académico encuestado expresó:

- Desconocer si se presentan

- No haber observado ninguna

- No haber percibido actitudes que demuestren falta de equidad

Es un aspecto con el que coinciden algunos estudiantes al expresar que no saben o desconocen sobre ello.

Al preguntarles si conocían si en la normatividad universitaria se contemplan políticas referentes a la equidad, un $25 \%$ de los encuestados aceptó desconocer si existen políticas referentes a equidad en la normatividad universitaria, debido a que no han leído dicha normatividad. Mientras que un $37.5 \%$ afirmó conocerlo de manera parcial. Solo un 25\% de los docentes afirmó conocer que sí existen políticas relativas a equidad en la normatividad de la Universidad Veracruzana y mencionan el Reglamento para la Equidad de Género y La Guía para la atención de casos de Hostigamiento y Acoso sexual. En cuanto al alumnado encuestado, un 18,05\% asegura conocer que sí se contemplan políticas referentes a la equidad en la normatividad universitaria, mientras que un $44,44 \%$ acepta que solo conoce parcialmente y un $37,5 \%$ acepta no conocerlo.

En cuanto a la normatividad que conocen, principalmente mencionaron:

- Reglamento para la equidad de género

- Guía para la atención de casos de hostigamiento y acoso sexual

Es interesante que los motivos de este desconocimiento que tanto docentes como alumnos mencionaron fueran:

- No haber leído la normatividad

- Falta de información

- Falta de difusión al respecto

- No prestar atención

- No recordarlo

Otro punto interesante de compartir es que cuando se les cuestionó si existía una cultura de calidad en la Facultad de Arquitectura, el 62,5\% de los docentes encuestados aseguró que sí existe una cultura de calidad en la Facultad de Arquitectura, el 25\% coincidió en que sí existe dicha cultura, pero solo parcialmente, debido a que no todos la practican. Cabe mencionar que ningún académico negó la existencia de una cultura de calidad en dicha Facultad. 
En cuanto a los estudiantes, solo un 19,44\% de estos opinaron que sí existe una cultura de calidad en la Facultad de Arquitectura, mientras que el 55,55\% de los estudiantes considera que existe solo de manera parcial y el 19,44\% restante fue tajante al considerar que no existe una cultura de calidad en la Facultad de Arquitectura.

Sobre los motivos por los que opinaban así, los docentes expresaron cuatro principales aspectos:

1. La formación del alumnado: se ha establecido que la calidad de la enseñanza es lo primero en la formación de los alumnos.

2. Índices de calidad institucional: académicos, alumnos y personas somos un equipo, realizamos una labor constante de calidad.

3. Ambiente: es un ambiente sano y ameno.

4. Cultura parcial de calidad: porque se ha desarrollado, pero no se ha trabajado en las recomendaciones, algunos la practican y otros no.

Los alumnos argumentaron su respuesta en los siguientes aspectos:

- No en todos los ámbitos existe

- Se debe mejorar algunas cosas

- Lo desconocen

- No se promueve

Sobre las características que una institución educativa debería tener para fomentar la equidad y la calidad, cabe mencionar que a excepción de «sancionar la falta de aplicabilidad de la normatividad» que obtuvo un $12,5 \%$ de rechazo, todas las demás características fueron mencionadas por los docentes como total o parcialmente aceptadas en el fomento de la equidad y calidad. Tal es el caso de la transparencia que obtuvo un 100\% de aceptación por parte de los docentes en el fomento de la equidad y la calidad. Le siguieron atención a la diversidad y pertinencia de los programas que obtuvieron un puntaje de $87,5 \%$. Mientras que una aplicación plena de la normativa y la sanción a la falta de su aplicación fueron totalmente aceptadas por un $75 \%$ de los académicos. El 62,5\% también considera totalmente necesaria la adecuación de espacios físicos y las adecuaciones curriculares en el fomento de la equidad y la calidad. Finalmente, con un 50\%, los docentes colocan a la vinculación de los programas interinstitucionales y a la difusión de las buenas prácticas como totalmente necesarias. Como se pudo observar, más del 50\% de los profesores reconocen como totalmente importantes todas las características enlistadas. 
Los alumnos, por su parte, en un 69,44\% consideran la atención a la diversidad, la transparencia con un $63,88 \%$ y la adecuación de espacios físicos con $62.5 \%$ como las tres características más importantes a atender. Mientras que un $6,9 \%$ consideran adecuaciones curriculares, pertinencia de los programas, la vinculación de los programas interinstitucionales como características que para nada deben estar para que en una institución educativa se cultive la equidad y la calidad.

Finalmente, nos interesó presentar la forma en que conceptualizan tanto docentes como alumnos a la equidad:

\section{Docentes:}

- Que se reciba y se impartan los conocimientos de manera equitativa.

- Apreciación de la dignidad de todos los seres humanos, más allá de sus particularidades, apariencias y circunstancias.

- Dar un trato equitativo a hombres y mujeres, no asumir que un sexo es superior a el otro, la cualidad de dar a cada persona lo que se merece sin exceder o disminuir sin favorecer, igualdad.

- Igualdad para todos en todos aspectos.

- Es el no ser parcial con alguna persona o grupo de personas en cualquier forma de tal manera que perjudique a otra.

- No favorecer o desfavorecer en trato a una persona por sexo, origen, edad, posición económica.

\section{Alumnos:}

- Igualdad, ante todo.

- Ser tratados por igual.

- Dar a cada quién lo que le corresponde.

- Respetar todo tipo de individuos o creencias.

- Que no se hagan distinciones.

- Proporcionar a las personas lo que necesitan para tener las mismas oportunidades.

- Ser considerados sin distinción de género o estatus social.

- Todos trabajamos según nuestras habilidades o aptitudes, pero bajo los mismos derechos u obligaciones. 


\section{Discusión}

Los resultados obtenidos nos permiten reflexionar hacia aspectos como que a pesar de que la Universidad Veracruzana (2016) cuenta con una normatividad en materia de equidad y calidad, todavía existe un atisbo de confusión o desconocimiento al respecto, tanto en el personal docente como en el alumnado, lo cual nos lleva a cuestionarnos sobre cuáles serán las principales razones de ello.

Otro elemento que consideramos necesario atender es el hecho de que tanto maestros como alumnos no poseen conocimiento preciso de cuáles son los ámbitos de competencia para la atención de la equidad en la Facultad de Arquitectura.

La cultura de la calidad institucional también se identifica con debilidad, característica básica a atender para cumplir con los índices de calidad tanto nacionales como internacionales.

En cuanto a las áreas de oportunidad, podemos citar la apertura y disposición tanto del personal académico como de los aprendientes para involucrarse y participar en la promoción de la equidad. Además de la existencia y colaboración del Observatorio Regional para la Calidad y Equidad en la Educación Superior (ORACLE) en la identificación de situaciones de inequidad y orientar a los actores para su atención, coadyuvando en el mejoramiento de la calidad educativa institucional.

\section{Conclusiones}

Desarrollamos las conclusiones en función del análisis de los tres apartados que integraron la encuesta. Iniciamos con el apartado Pertinencia de las políticas institucionales: La mayoría del personal docente y del estudiantado considera importante la promoción de políticas de equidad en la Facultad de Arquitectura como el manejo de la equidad institucional con respecto a la calidad de la educación que se imparte en la Universidad Veracruzana. Sin embargo, en algunos de los estudiantes aún existe indiferencia respecto a la relación existente entre equidad y calidad además de que existe en parte del estudiantado confusión entre los términos equidad e igualdad. Otro aspecto importante es el hecho de que un número significativo tanto de docentes como de estudiantes externaron desconocer o conocer parcialmente las políticas de equidad de la institución, aspecto que en cierta manera se relaciona con la confusión conceptual antes mencionada y con no relacionar la atención a la equidad con el nivel de calidad de la institución. 
A continuación, presentamos el análisis del segundo apartado, Detección de necesidades y problemáticas: Definitivamente las miradas de ambos estamentos (docentes y alumnos) coinciden en la identificación de problemáticas como: Prepotencia/abuso del poder, problemáticas de las relaciones entre maestros, la forma de relacionarse entre maestros y personal administrativo, además, algunos estudiantes identificaron problemáticas como: acoso y maltrato verbal. Aspectos que se vuelven en un área de oportunidad a atender.

En relación con tercer apartado, opiniones de los encuestados, con respecto a la operatividad de la normativa, promoción de la equidad en la institución, estrategias que se implementan y el desarrollo de la cultura de la calidad educativa: pudimos observar que las prácticas de equidad en la Facultad de Arquitectura son consideradas apropiadas. En cuanto a valores a fomentar en la U.V., se mencionan principalmente tolerancia, respeto a la diversidad de ideas, igualdad y no discriminación, libertad, ética profesional, y respeto a la diversidad sexual, destacamos el hecho de que el 63,8\% de los alumnos coinciden en elegir a la equidad como es el valor más necesario de cultivar. En relación a la normatividad universitaria y políticas referentes a la equidad, aún existe desconocimiento, sin embargo, un porcentaje mayor afirmó conocer que sí existen políticas relativas a equidad en la normatividad de la Universidad Veracruzana siendo el Reglamento para la Equidad de Género y La Guía para la atención de casos de Hostigamiento y Acoso sexual los más mencionados. Para cerrar este apartado, es interesante mencionar que parte significativa de la población encuestada considera que sí existe una cultura de calidad en la Facultad de Arquitectura en mayor o menor medida, pero existe.

Indiscutiblemente, a nuestro parecer existe una relación entre las prácticas de equidad de una institución educativa con la calidad que esta ofrece. En este sentido, la Facultad de Arquitectura de la Universidad Veracruzana, en su región Poza Rica - Tuxpan tiene un reto a enfrentar: mejorar sus prácticas de equidad a fin de elevar el nivel de calidad que ofrece.

Sin embargo, tal tarea no es fácil pues requiere del involucramiento de todos y cada uno de los agentes participantes. Siendo una de las primeras tareas trabajar en la conceptualización que se tiene acerca de la equidad, evitando reducirla solo a lo que género se refiere.

Finalmente, consideramos necesario trabajar en lo que a cultura de calidad se refiere, sobre todo a la imagen que tienen los estudiantes al respecto. Por lo que sus prácticas deberán apegarse a la normatividad institucional a fin de cumplir con lo establecido. 


\section{REFERENCIAS BIBLIOGRÁFICAS}

Calvo, M. (2011) Educando para la igualdad. Pamplona, España: EUNSA.

Facultad Latinoamericana de Ciencias Sociales (Flacso) (2017). Integración, equidad y desarrollo. Quito, Argentina: Flacso.

Gairín, S. J., Castro, D., Rodríguez, G. D. y Barrera, A. (2015). Acceso, permanencia y éxito académico de colectivos vulnerables en la Educación Superior. Estrategias para la intervención. Barcelona, España: EDO-SERVEIS, Universidad Autónoma de Barcelona.

Gairín, S. J. y Suárez, C. I. (2018). Equidad y Educación Superior. En S. J. Gairín, Politicas y prácticas para la equidad en la Educación Superior. Madrid, España: Wolters Kluwer.

Granados, O. (2018) Reforma educativa. México, D.F.: FCE.

Hernández, R., Fernández, C., Baptista, P. (2010) Metodología de la Investigación. 5a. ed. México, D.F.: Mc Graw Hill / Interamericana Editores, S.A. DE C.V.

Hernández, R. A. y Coello, Z. (2008) El paradigma cuantitativo de la investigación científica. La Habana, Cuba: Editorial Universitaria.

Llach, J. J. (2010) El desafío de la equidad educativa: diagnóstico y propuestas. Buenos Aires, Argentina, Argentina: Ediciones Granica.

Ley Orgánica de la Universidad Veracruzana (2017). Consultado el 14 de junio de 2019. Recuperado de https://www.uv.mx/legislacion/files/2019/04/ Ley-Organica-Universidad-Veracruzana-reimpresion2017.pdf

NU. CEPAL OEA (1995) Educación, eficienciay equidad. Editadopor Ernesto Cohen. Capítulo2. Eldesafío educativo: en busca dela equidad perdida. Hopenhayn, Martín. Editorial CEPAL, OEA, Ediciones Sur. Recuperado de https:// www.cepal.org/es/publicaciones/1762-educacion-eficiencia-equidad

Ricaurte, Q. C., Albuquerque, M. A., Granda, G., Jerónimo, M., Jiménez, S. F., Lopes, R. M. y Vázquez, C. M. (2018). Diagnóstico de equidad en las funciones universitarias. En S. J. Gairín, Políticas y prácticas para la equidad en la Educación Superior (p. 327). Madrid, España: Wolters Kluwer.

Rubio Oca, J. y Mungaray, A. (2009) El reto de la equidad en la educación mexicana. México, D.F. México: Porrúa.

Santos, M.A. (2013) Políticas educativas y compromiso social: el progreso de la equidad y la calidad. Barcelona, Espańa: Octaedro, S.L.

Unesco (2017) Informe de Seguimiento de la Educación en el Mundo 2016. La educación al servicio de los pueblos y el planeta: creación de futuros sostenibles para todos. Recuperado de https://gem-report-2016.unesco. org/es/chapter/meta-4-5-equidad/ 
Universidad Veracruzana (2016). Código de Ética. Recuperado de https://www. uv.mx/legislacion/files/2017/07/Codigo-de-etica-de-la-UniversidadVeracruzana.pdf

Universidad Veracruzana (2020) Sitio web. Sección Nosotros/Presentación. Recuperado de https://www.uv.mx/universidad/presentacion/

Yanes, J. (2015) Complejidad y calidad de la educación. Santiago de Chile: RIL Editores.

Zabalza, M. A. (2016) Calidad en la educación infantil. Madrid, España: Narcea Ediciones. 